\title{
Thermal fatigue on pistons induced by shaped high power laser. Part II: Design of spatial intensity distribution via numerical simulation
}

\author{
Hong-Wei Song ${ }^{\mathrm{a}, *}$, Gang Yu ${ }^{\mathrm{a}}$, Alexander F.H. Kaplan ${ }^{\mathrm{b}}$, Jian-Song Tan ${ }^{\mathrm{c}}$, Xiao-Li Yu ${ }^{\mathrm{c}}$ \\ ${ }^{a}$ Laboratory for Laser Intelligent Manufacturing, Institute of Mechanics, Chinese Academy of Sciences, Beijing 100080, PR China \\ ${ }^{\mathrm{b}}$ Division of Manufacturing Systems Engineering, Luleå University of Technology, SE-97187 Luleå, Sweden \\ ${ }^{\mathrm{c}}$ The College of Mechanical and Energy Engineering, Zhejiang University, Hangzhou 310027, PR China
}

Received 2 February 2006; received in revised form 14 April 2007

Available online 5 July 2007

\begin{abstract}
In the laser induced thermal fatigue simulation test on pistons, the high power laser was transformed from the incident Gaussian beam into a concentric multi-circular pattern with specific intensity ratio. The spatial intensity distribution of the shaped beam, which determines the temperature field in the piston, must be designed before a diffractive optical element (DOE) can be manufactured. In this paper, a reverse method based on finite element model (FEM) was proposed to design the intensity distribution in order to simulate the thermal loadings on pistons. Temperature fields were obtained by solving a transient three-dimensional heat conduction equation with convective boundary conditions at the surfaces of the piston workpiece. The numerical model then was validated by approaching the computational results to the experimental data. During the process, some important parameters including laser absorptivity, convective heat transfer coefficient, thermal conductivity and Biot number were also validated. Then, optimization procedure was processed to find favorable spatial intensity distribution for the shaped beam, with the aid of the validated FEM. The analysis shows that the reverse method incorporated with numerical simulation can reduce design cycle and design expense efficiently. This method can serve as a kind of virtual experimental vehicle as well, which makes the thermal fatigue simulation test more controllable and predictable.
\end{abstract}

(C) 2007 Elsevier Ltd. All rights reserved.

Keywords: High power laser; Beam shaping; Temperature field; Thermal fatigue; Finite element

\section{Introduction}

Thermal fatigue simulation test on pistons based on the shaped high power laser is a novel conception. In this conception, the incident Gaussian beam was transformed into concentric multi-circular beam with specific intensity distribution, in order to induce the transient temperature fields on the top surface of piston, similar to those in service [1]. Laser is usually characterized by extreme focusing and directional irradiation at high energy density [2]. However, the temperature field on the top surface of the piston

\footnotetext{
* Corresponding author. Tel.: +86 010 62651165; fax: +86 010 62561284.

E-mail address: songhw@imech.ac.cn (H.-W. Song).
}

is as large as $150 \mathrm{~mm}$ in diameter. The laser beam must be modulated in the spatial domain to cover such a large range.

Laser beam shaping, the control of the irradiance and phase profile of the output of a laser, is an enabling technology used in a number of industrial sectors $[3,4]$. The spatial shaping of laser beam is the process of redistributing the irradiance of laser. Diffractive optical elements (DOEs) can modulate freely the irradiance and phase profile of laser beam using their surface relief microstructures [5]. A very common beam shaping requirement is a laser profile that is uniform over some cross-section $[3,6]$, which is the case of the present study. Usually the intensity and phase distribution of a DOE is optimized by a method based on fast Fourier transform (FFT) calculation and 


\section{Nomenclature}

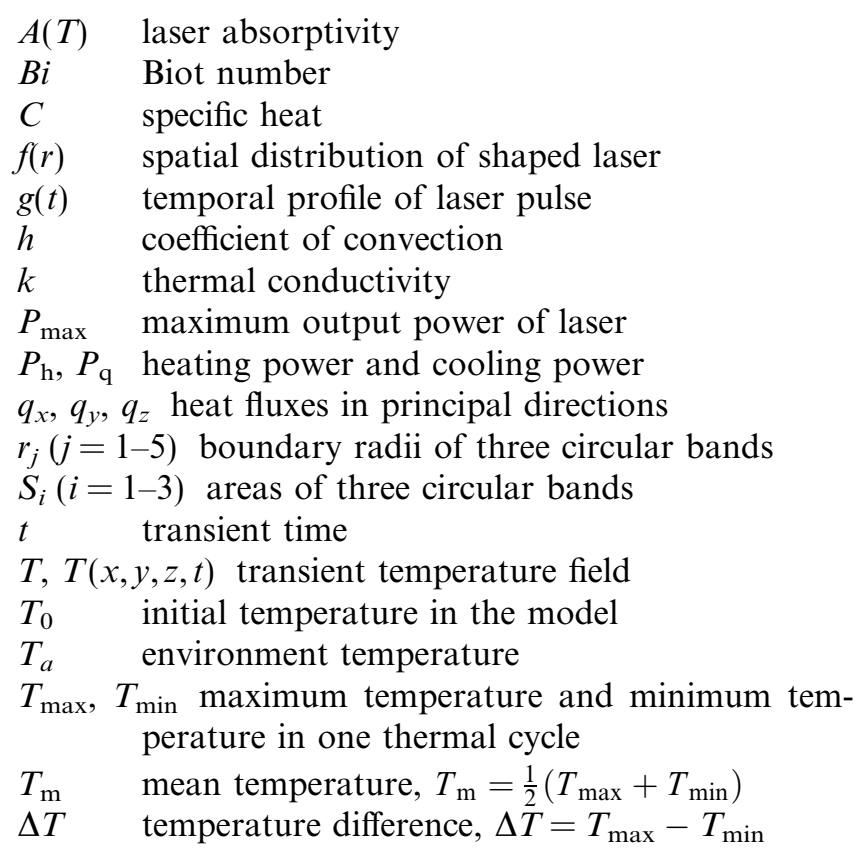

\author{
Greek symbols \\ $\delta_{i}(i=1-3)$ ratio of laser irradiance between three circu- \\ lar bands \\ $\rho \quad$ material density \\ $\tau_{\mathrm{h}}, \tau_{\mathrm{q}}$ heating duration and cooling duration in one \\ thermal cycle \\ $\nabla^{2} \quad$ Laplacian operator

\begin{tabular}{ll}
\multicolumn{2}{l}{ Subscripts } \\
0 & initial \\
$\mathrm{h}$ & heating \\
$\mathrm{m}$ & mean \\
$\max$ & maximum \\
$\mathrm{min}$ & minimum \\
$\mathrm{q}$ & cooling
\end{tabular} \\ Subscripts \\ initial \\ h heating \\ mean \\ $\max$ maximum \\ q cooling
}

iterative processes. Then, the optimal DOE is precisely fabricated by photolithography and plasma etching process.

The intensity distribution of shaped laser beam for obtaining desired thermal property can be mathematically calculated in simple cases. However, it is not the case in the present study, due to the complexity of piston geometry. Therefore, we proposed a reverse method incorporated with numerical analysis to find the pertinent solution. To achieve this goal, an effective finite element model (FEM) should be built first. Then, the optimal design for intensity distribution of shaped beam transformed from DOE could be obtained through iterative process.

\section{Outline of the general method}

As mentioned in the accompanying paper [1] and documented in the experimental report [7], the temperatures of center, middle and outer circular bands on the top surface oscillate in the ranges of $310-320^{\circ} \mathrm{C}, 280-290{ }^{\circ} \mathrm{C}, 350$ $360^{\circ} \mathrm{C}$, respectively, during a cooling and heating cycle. This composes the target temperature field for DOE design. One must find the right intensity distribution for the shaped beam to produce this transient target temperature field.

The reverse method based on finite element analysis is illustrated in the flowchart of Fig. 1. Two major steps were incorporated in this method. The first step is validation of FEM. A FEM that is accorded with the actual loading condition of an originally designed DOE was established first. Then, the FEM was validated by approaching the computational results to the sampled temperatures in characteris- tic areas. During this process, the influence of some important parameters, including laser absorptivity, convective heat transfer coefficient, thermal conductivity and Biot number, were also validated and discussed. The second step is optimization of spatial intensity distribution. With the validated FEM, the target temperature fields and temperature oscillations were achieved by adjusting the intensity field and loading case. An updated DOE could therefore be manufactured to transform the laser beam into the optimal intensity distribution.

\section{Finite element modeling}

Absorption takes effect when the shaped laser irradiated on the top surface of the piston. Absorption is the mechanism generating a certain amount of heat from the optical energy of the laser radiation, while the remaining portion is either reflected or transmitted [8]. It takes place in a surface layer typically in the order of $30-70 \mathrm{~nm}$ in thickness $[9,10]$. Meanwhile, processes of convection at the surfaces and conduction in the workpiece would determine the temperature field.

Although the piston is a cylindrical structure, it is not axisymmetric, therefore Cartesian coordinate system instead of cylindrical coordinate system was employed while modeling. The piston has a symmetry plane of $x-z$ however, and a 3D FEM was built for the $1 / 2$ solid structure, in order to reduce computational expense. The model is illustrated in Fig. 2. Meshes of laser irradiated regions on the top surface were refined to obtain good accuracy, as is shown in Fig. 3. Refer to Fig. 4 and also Fig. 3, the shaped 


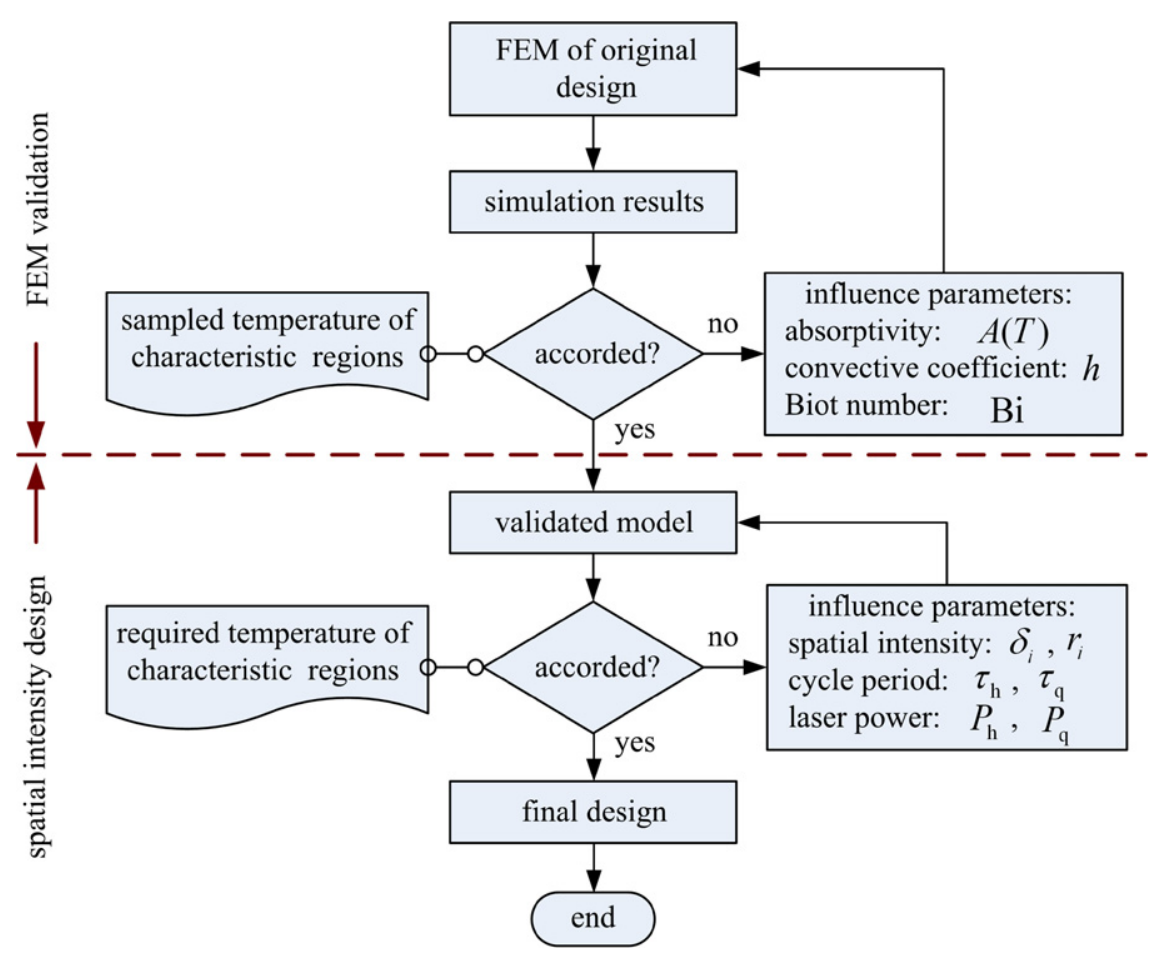

Fig. 1. Flowchart of FEM-based design for spatial intensity distribution of the shaped laser.

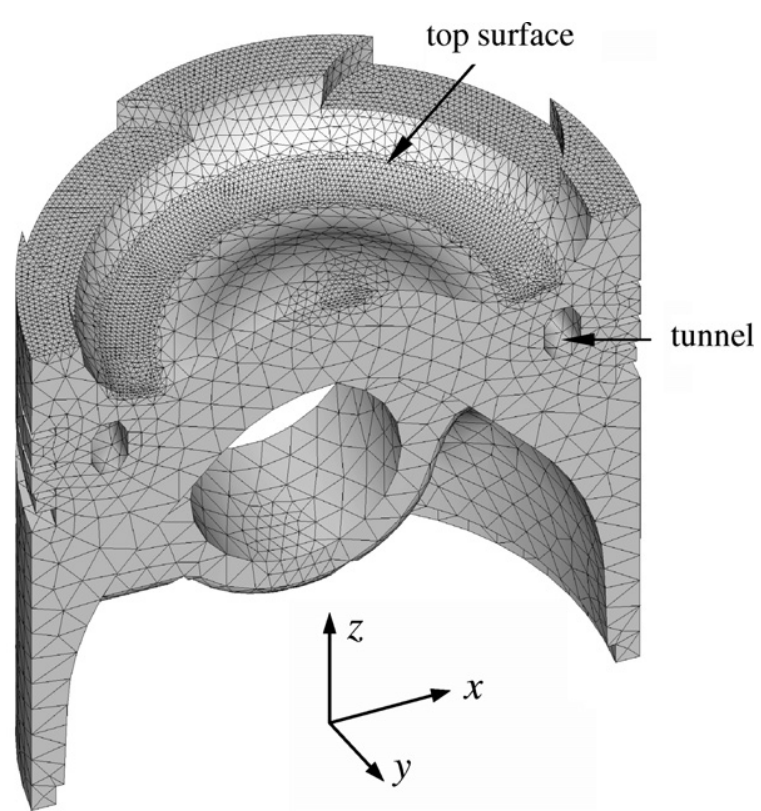

Fig. 2. 3D FEM of a half piston.

beam irradiated on the top surface distributes into three concentric circular bands. The spatial intensity distribution can be written into

$$
f(r)= \begin{cases}\delta_{1}, & r \in\left[0, r_{1}\right] \\ \delta_{2}, & r \in\left[r_{2}, r_{3}\right] \\ \delta_{3}, & r \in\left[r_{4}, r_{5}\right] \\ 0, & r \in\left[r_{1}, r_{2}\right] \cup\left[r_{3}, r_{4}\right] \cup\left[r_{5}, \infty\right]\end{cases}
$$

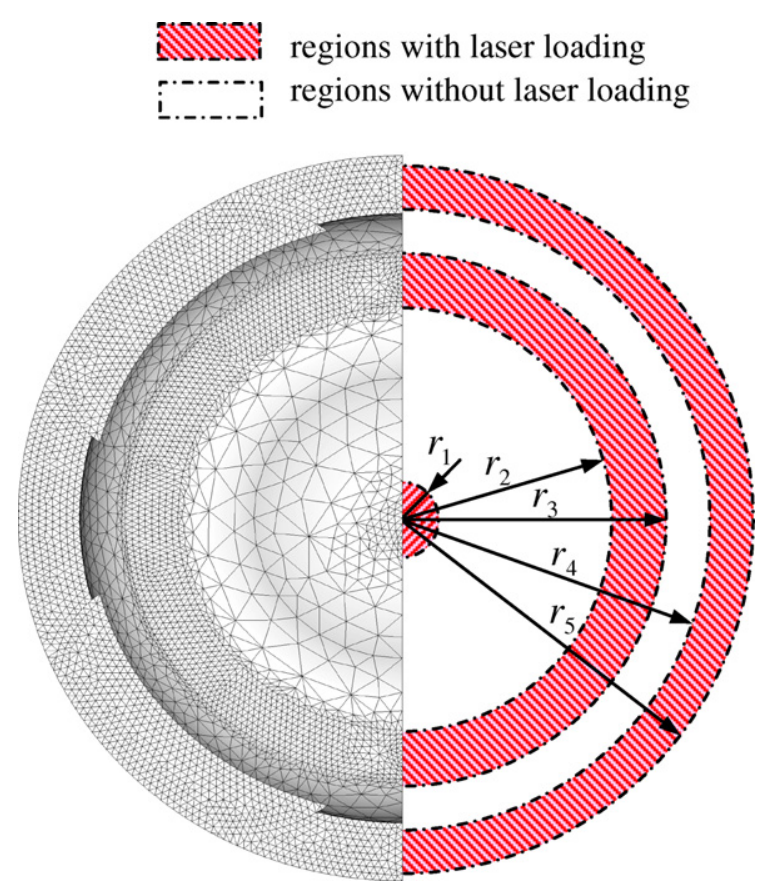

Fig. 3. Pattern of shaped beam and fine meshes at top surface.

where $\delta_{i}(i=1-3)$ are the ratios of laser irradiance between center circular band, middle circular band and outer circular band, and $r_{j}(j=1-5)$ are boundary radii of the three circular bands. Radius $r$ could be calculated from $r=\sqrt{x^{2}+y^{2}}$. The ultimate goal now turns to find optimum values of $\delta_{i}$ and $r_{j}$ in Eq. (1), in order to satisfy temperature distribution on the top surface of the piston. 


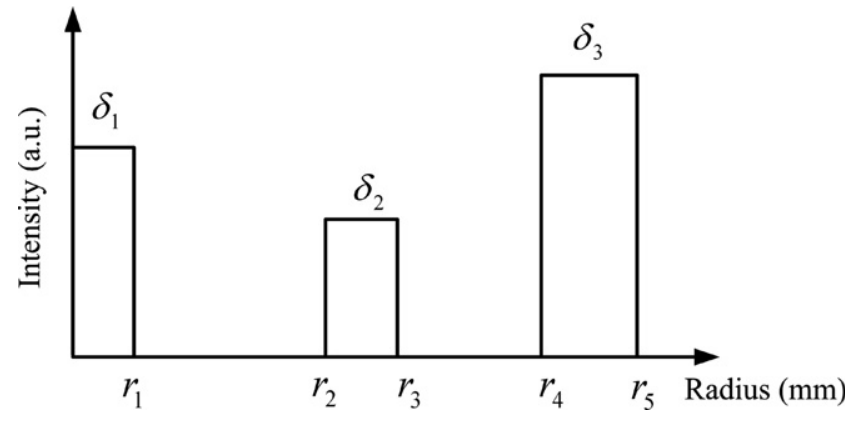

Fig. 4. Spatial intensity distribution of shaped beam.

Suppose the piston material is homogenous, therefore the coefficient of thermal conductivity is equal in each principal direction, the transient temperature field $T(x, y, z, t)$ in the piston volume satisfies differential equation [11]

$\rho C \frac{\partial T}{\partial t}-k \nabla^{2} T=0$

where $\rho, C$ and $k$ are the density, specific heat and thermal conductivity, respectively. The Laplacian $\nabla^{2}$ in the Cartesian coordinates is $\frac{\partial^{2}}{\partial x^{2}}+\frac{\partial^{2}}{\partial y^{2}}+\frac{\partial^{2}}{\partial z^{2}}$. The top surface of the piston was irradiated by the shaped laser, and the instantaneous boundary heat flux obeys Fourier's law of heat conduction

$q_{z}=-k \frac{\partial T}{\partial z}=A(T) I_{0} f(r) g(t)$

where $A(T)$ is the laser absorptivity, which is defined as the ratio of the power absorbed at the surface to the incident power at temperature $T ; I_{0}$ is the unit laser irradiance of the shaped beam; $f(r)$ is the spatial distribution of shaped laser which obey Eq. (1), $g(t)$ is the temporal profile of the laser pulse. The temporal profile $g(t)$ may be in diverse forms, taking square pulses as an example (which is normally used in the present numerical model), it can be written into

$$
g(t)=\left\{\begin{array}{l}
P_{\mathrm{h}} / P_{\max }, \\
t \in\left[i \tau_{\mathrm{h}}+i \tau_{\mathrm{q}},(i+1) \tau_{\mathrm{h}}+i \tau_{\mathrm{q}}\right] \\
P_{\mathrm{q}} / P_{\max }, \\
t \in\left[(i+1) \tau_{\mathrm{h}}+i \tau_{\mathrm{q}},(i+1)\left(\tau_{\mathrm{h}}+\tau_{\mathrm{q}}\right)\right]
\end{array} \quad(i=0,1,2 \ldots . . n)\right.
$$

where $\tau_{\mathrm{h}}$ and $\tau_{\mathrm{q}}$ are the heating duration and cooling duration in one thermal cycle, respectively, $i$ is the number of pulses; $P_{\mathrm{h}}$ and $P_{\mathrm{q}}$ are the laser powers at heating and cooling stages, respectively. $P_{\max }$ is the maximum output power of laser, in the present case, $P_{\max }$ equals $3000 \mathrm{~W}$. The temporal profile is illustrated in Fig. 5. The laser power at cooling stage, $P_{\mathrm{q}}$, may equal zero, and under this condition the heat flux on the top surface is

$q_{z}=-k \frac{\partial T}{\partial z}=0$

The unit laser irradiance is obtained by

$I_{0}=\frac{P_{\max }}{\sum \delta_{i} S_{i}}$

where $S_{i}(i=1-3)$ are the areas irradiated by the shaped beam, $S_{1}, S_{2}$ and $S_{3}$ represent the areas of center, middle and outer circular bands, respectively. Refer to Fig. 3, one can obtain $S_{1}=\pi r_{1}^{2}, \quad S_{2}=\pi\left(r_{3}^{2}-r_{2}^{2}\right), \quad$ and $S_{3}=$ $\pi\left(r_{5}^{2}-r_{4}^{2}\right)$.

Surfaces besides the plane of symmetry and the bottom surface exchange heat with environment. According to convective heat transfer boundary condition $\left(\Gamma_{\mathrm{III}}\right.$-type boundary), one gets

$-\left.k \frac{\partial T}{\partial n}\right|_{\Gamma_{\mathrm{III}}}=h\left(T-T_{a}\right)$

where $h$ is the coefficient of convective heat transfer, $T_{a}$ is the environmental temperature, $n$ represents normal direction to the surface. Unlike the working condition of the engine, the piston is settled on the workbench where the surrounding environmental temperature keeps constant, therefore, spatial variation in the heat transfer coefficient over the structure can be neglected.

Thermal insulation is applied to the plane of symmetry and bottom surface, therefore

$-\left.k \frac{\partial T}{\partial n}\right|_{S}=0$

All elements were put an initial temperature at time $t=0$,

$T(x, y, z, 0)=T_{0}$

which denotes the end of warm-up preparation and the beginning of thermal fatigue test.

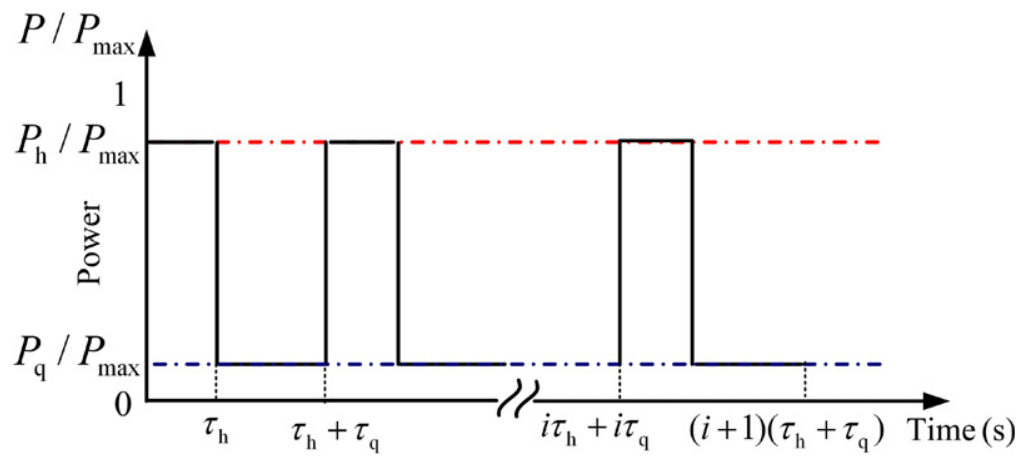

Fig. 5. Temporal profile of laser pulses, taking square pulses as an example. 


\section{Model validation}

Computational results of transient temperature distribution and fluctuation are dictated by several important factors, including heat capacity absorbed by the structure, the heat transfer condition over the surface of the body, thermal property of material, and overall shape of the structure, etc. These factors influence thermal stress and thermal fatigue in the structure as well [12]. However, laser absorptivity, convective heat transfer coefficient and thermal conductivity cannot be obtained readily from referential books. Reasonable values for these parameters must be determined before a reliable analysis could be carried out. Indeed, the process of model validation is to find the true parameters.

\subsection{Laser absorptivity}

The absorptivity $A(T)$ at the surface is the percentage of optical energy converted to heat. Absorptivity as the portion of heat generated from the laser beam energy hitting a surface essentially not only determines the process efficiency but the whole process [10]. To calculate the temperature field numerically in laser surface heating processes, laser beam absorptivity should be determined first. However, it is extremely difficult to find an accurate value for laser beam absorptivity $[10,13]$. The absorptivity depends on the wavelength, material and temperature, and it is also affected by the surface condition of the material and angle of incidence of the beam [14-16]. Absorptivity can be augmented by applying an absorptive coating to the surface, or by allowing the surface to oxidize during processing [17].

Mathematical models $[10,18,19]$ and experimental procedures [13] are the two basic methods in determining the absorptivity. In the present case, it is extremely difficult to find the accurate absorptivity either experimentally or theoretically, due to the complexity of piston surface topography and unknown property of surface coating. The wavelength of radiation from the Nd:YAG laser, which is utilized in the present study [1], is $1.064 \mu \mathrm{m}$, and it is absorbed more readily by metals than longer wavelength $\mathrm{CO}_{2}$ laser radiation. Meanwhile, the variation of absorptivity with temperature is not as significant as that found with $\mathrm{CO}_{2}$ laser radiation [17]. The temperature in the aluminum alloy piston vary only among $280-360{ }^{\circ} \mathrm{C}$ during the thermal cycles, therefore, a fixed absorptivity was applied in the numerical model. Referential values of 0.3-0.4 were documented for aluminum alloy to absorb $1.064 \mu \mathrm{m}$ radiation. A numerical validation was carried out to find the more reasonable value.

Fig. 6 shows the influence of absorptivity on the mean temperature and temperature difference of three laser irradiated bands (i.e., center, middle and outer circular bands). The data were sampled from numerical results after calculation of 30 thermal cycles. Suppose $T_{\max }$ and $T_{\min }$ are the maximum temperature and minimum temperature of the sampling area in one thermal cycle, respectively, the mean

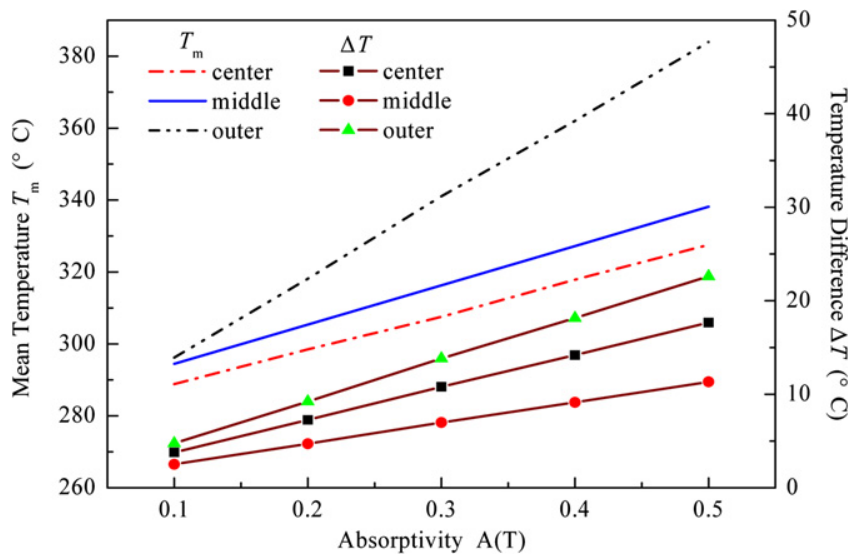

Fig. 6. Influence of $A(T)$ on $T_{\mathrm{m}}$ and $\Delta T$.

temperature, which indicates the temperature level of the sampling area, can be calculated from

$T_{\mathrm{m}}=\frac{1}{2}\left(T_{\max }+T_{\min }\right)$

and the temperature difference, which is the magnitude of temperature oscillation in one thermal cycle, is defined as

$\Delta T=T_{\max }-T_{\min }$

Increase in absorptivity means augmentation of input heat capacity in the computational model. Therefore, the increase of overall temperature in the piston structure is predictable. Fig. 6 also indicates that temperature gradient is proportional to absorptivity, as the evidence the difference of mean temperature $T_{\mathrm{m}}$ between center band and outer band raises from $10{ }^{\circ} \mathrm{C}$ at $A(T)=0.1$ to $70^{\circ} \mathrm{C}$ at $A(T)=0.5$. Meanwhile, temperature difference $\Delta T$ of each band raises dramatically with $A(T)$. When approaching experimental results, we found that $A(T)=0.3$ predicts temperature field and temperature difference more accurately.

\subsection{Convective heat transfer coefficient, thermal conductivity and Biot number}

Convective heat transfer is the function of temperature field and locations. Because of various complexities of the convective process, only very simple problems can be solved by the available analytical methods, whereas experimental methods are time consuming and very expensive. Therefore, one must resort to numerical methods for obtaining the desired information [20]. The actual value of the conductivity greatly influences the magnitude of the temperature differences and so controls the magnitude of the resulting thermal stresses [21]. In the present transient state problem, heat transfer seeks to balance the external resistance due to the convection process at the surface and the internal resistance due to conduction in the material. Therefore, the dimensionless Biot number $B i$, which connects convection with conduction, was introduced to 


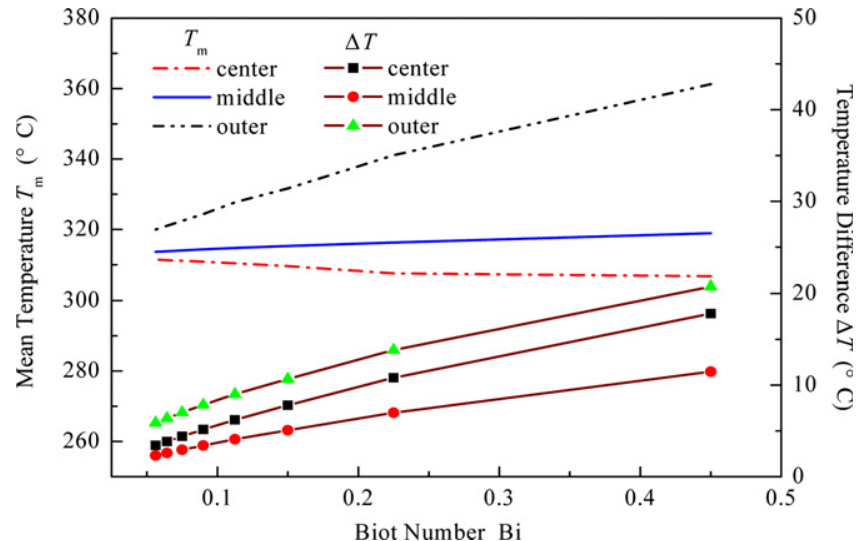

Fig. 7. Variation of $T_{\mathrm{m}}$ and $\Delta T$ with Biot number, with a fixed $h=60 \mathrm{~W} / \mathrm{mK}$.

validate the numerical model. The Biot number $(B i)$ is defined as $h L / k$, where $L$ is characteristic length.

Fig. 7 shows the influence of $B i$ on $T_{\mathrm{m}}$ and $\Delta T$ when the convective coefficient is fixed. The characteristic length $L$ was taken as the diameter of the piston. Thermal conductivity of aluminum alloy may range from 30 to $160 \mathrm{~W} / \mathrm{mK}$, depending on its composition [22]. With the fixed convective coefficient, $B i$ increases from 0.056 to 0.45 when the thermal conductivity drops from 160 to $20 \mathrm{~W} / \mathrm{mK}$. Temperature gradient increases significantly with $B i$, whereas the overall temperature does not increase that obviously. If the Biot number is small, heat transfer to the environment will have greater resistance, so the temperature in the solid will be roughly uniform. This analysis also indicates that material of high thermal conductivity should be selected for pistons, in order to avoid extreme high temperature gradient and thermal stress.

Fig. 8 shows the influence of $B i$ on $T_{\mathrm{m}}$ and $\Delta T$ when the thermal conductivity is fixed. The overall temperature drops dramatically when the Biot number increases, since the convection effect becomes significant. However, tem-

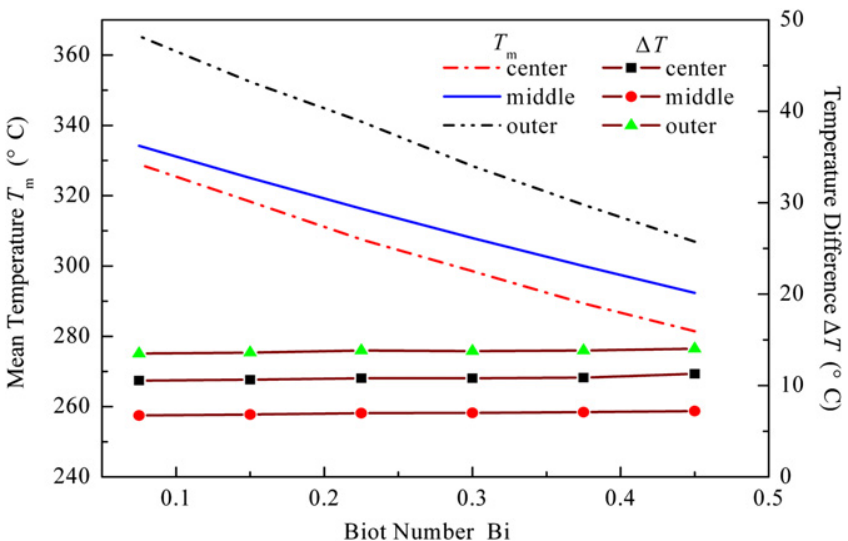

Fig. 8. Variation of $T_{\mathrm{m}}$ and $\Delta T$ with Biot number, with a fixed $k=40$ $\mathrm{W} / \mathrm{mK}$.

perature difference maintains relative even. In Fig. 7 the variable is thermal conductivity, whereas the variable in Fig. 8 is convective coefficient. Combining these two figures, it can conclude that the thermal conductivity dictates temperature gradient and temperature difference, whereas the convective coefficient dictates the temperature level of the structure.

\subsection{Model verification}

The numerical model was verified by a formerly manufactured DOE, by which the incident laser was shaped into an intensity field of $\delta_{1}=32, \delta_{2}=29, \delta_{3}=37$; and $r_{1}=5$, $r_{2}=40, r_{3}=50, r_{4}=60$ and $r_{5}=67.5 \mathrm{~mm}$. The loading case is periodic square pulses, with the heating power $P_{\mathrm{h}}=3000 \mathrm{~W}$ and heating duration $\tau_{\mathrm{h}}=2 \mathrm{~s}$, and cooling power $P_{\mathrm{q}}=0 \mathrm{~W}$ and cooling duration $\tau_{\mathrm{q}}=3 \mathrm{~s}$ in one thermal cycle. The initial temperature of $T_{0}=280{ }^{\circ} \mathrm{C}$ was assigned to the numerical model. Fig. 9 gives the comparison of computational and experimental results. It is found that the overall temperature increase tendency,
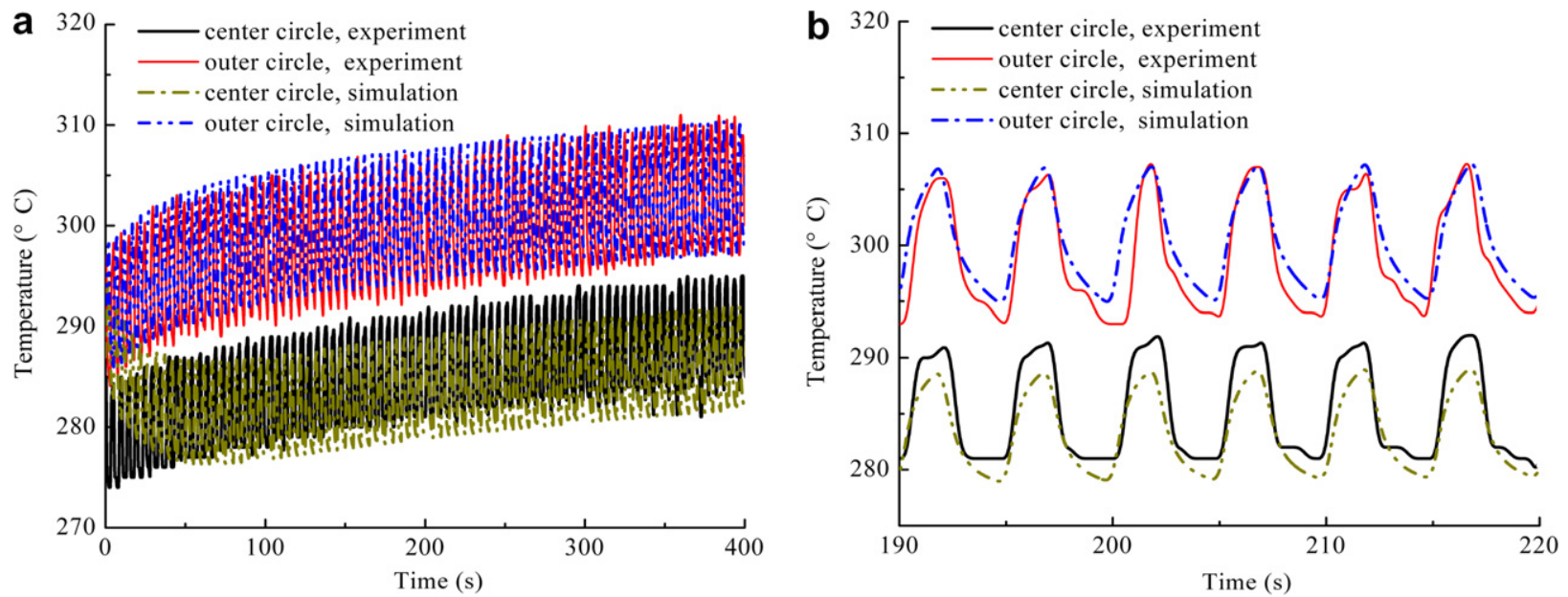

Fig. 9. Comparison of numerical and experimental result, with the original design: (a) 80 cycles; (b) selected 6 cycles. 


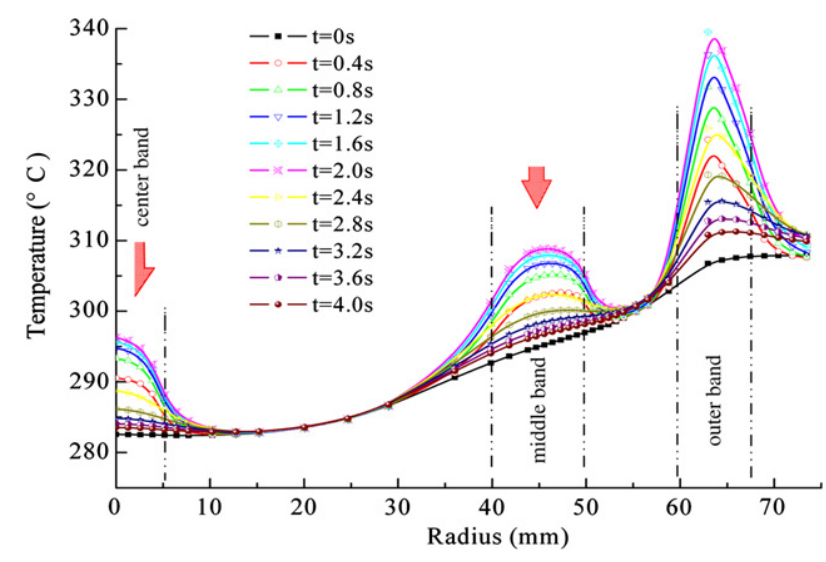

Fig. 10. Instantaneous temperatures across the radius during one thermal cycle, with the original design.

temperature gradient, and magnitude of temperature oscillation in each band obtained from the computational results are in good agreements with those obtained from experiments. The model was also validated by other loading cases of the same DOE, not listed for space saving.

Fig. 10 illustrates the instantaneous temperatures across the piston radius during one thermal cycle, calculated from another loading case of $P_{\mathrm{h}}=3000 \mathrm{~W}$ and $P_{\mathrm{q}}=0 \mathrm{~W}$, $\tau_{\mathrm{h}}=\tau_{\mathrm{q}}=2 \mathrm{~s}$. The laser irradiated bands can be distinguished from the magnitude of temperature difference between $t=2.0 \mathrm{~s}$ and $t=0 \mathrm{~s}$. It is observed that the temperature in the middle band is much higher than the target temperature, and the outer band is too close to the middle band.

\section{Optimal design and discussions}

\subsection{Intensity field optimization and design verification}

The second step, i.e., the optimization procedure illustrated in Fig. 1, was therefore carried out to find more favorable intensity distribution. The original design and the final optimal design are compared in Fig. 11, in the
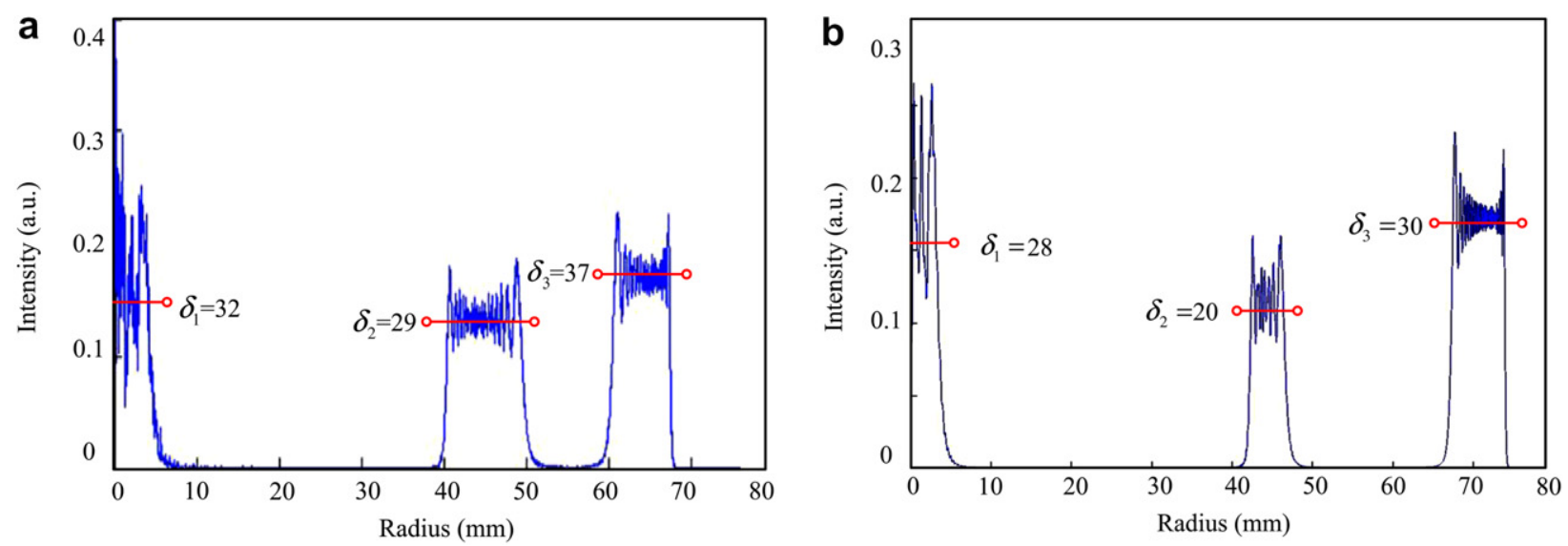

Fig. 11. Spatial intensity distribution of DOE shaped beam (a) original design; (b) optimal design.
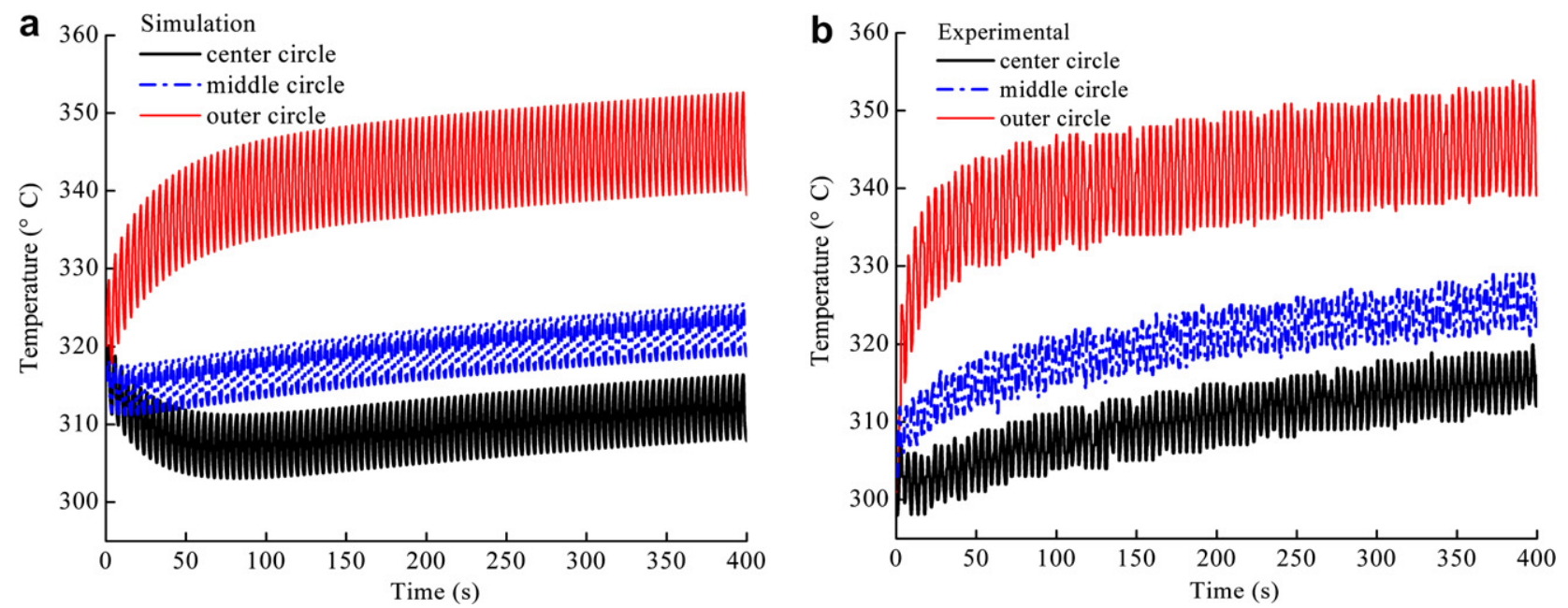

Fig. 12. Comparison of instantaneous temperature field between numerical and experimental, with the optimal design (a) simulation; (b) experimental. 
mode of intensity distribution calculated from the DOE microstructure pattern. Compared with the original design, the magnitude of intensity and dimension of the middle band was reduced, and the outer band was moved outwards. The optimal design is $\delta_{1}=28, \delta_{2}=20, \delta_{3}=30$, and $r_{1}=4, r_{2}=42, r_{3}=47, r_{4}=67$ and $r_{5}=74 \mathrm{~mm}$.

Again, Fig. 12 gives the verification of the computational result in the form of instantaneous temperature field. The periodic loading is $P_{\mathrm{h}}=2500 \mathrm{~W}$ and $P_{\mathrm{q}}=500 \mathrm{~W}$, $\tau_{\mathrm{h}}=\tau_{\mathrm{q}}=2 \mathrm{~s}$, and the initial temperature in the computational model is $T_{0}=310^{\circ} \mathrm{C}$. The temperature level and the magnitude of temperature oscillation in each band agree well with those obtained from experiments.

\section{2. $3 D$ transient temperature fields}

The advantage of simulation over experiment is that it can depict three-dimensional transient temperatures. One
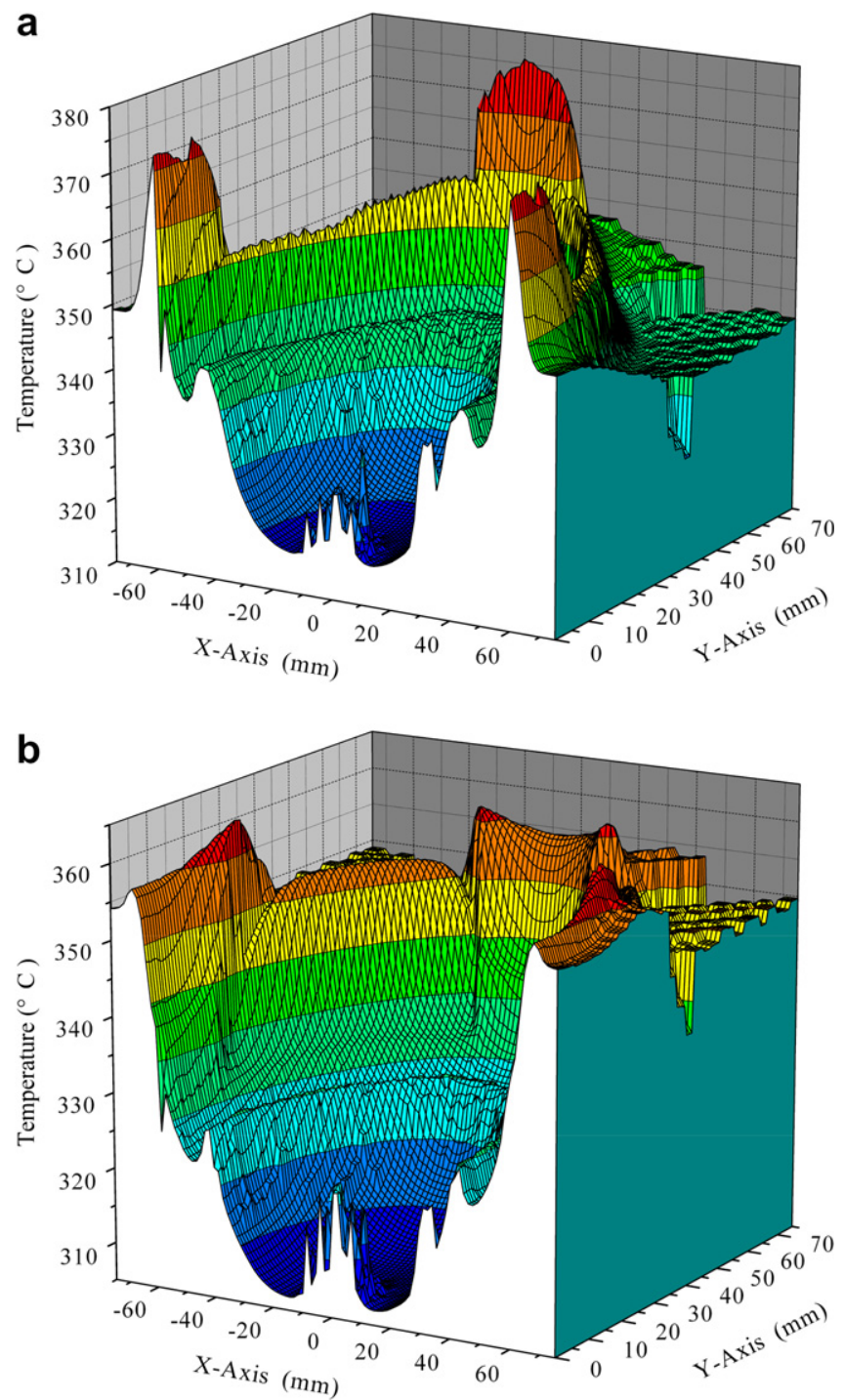

Fig. 13. Temperature field on the top surface of the piston (a) original design; (b) optimal design. can obtain the temperature information of any desired time for any desired location from the numerical result. Fig. 13 shows the temperature fields on the top surface of the piston at $t=398 \mathrm{~s}$, when both the original design and optimal design were subject to the periodic loading of $P_{\mathrm{h}}=2500 \mathrm{~W}$, $P_{\mathrm{q}}=500 \mathrm{~W}$ and $\tau_{\mathrm{h}}=\tau_{\mathrm{q}}=2 \mathrm{~s}$. If the temperatures were projected onto the $x-y$ plane, the profile of piston top surface could roughly be seen. Fig. 14 shows the instantaneous temperatures across the radius during 5 periodic thermal cycles from $t=380 \mathrm{~s}$ to $t=400 \mathrm{~s}$. It is found that the temperatures in the laser irradiated areas oscillate significantly, whereas little temperature variation was observed in the areas which are away from the irradiated bands. Fig. 13b and $14 \mathrm{~b}$ also indicate a significant drop in temperature in the middle band, and the temperature distribution in the outer band is more uniform, for the optimal design.
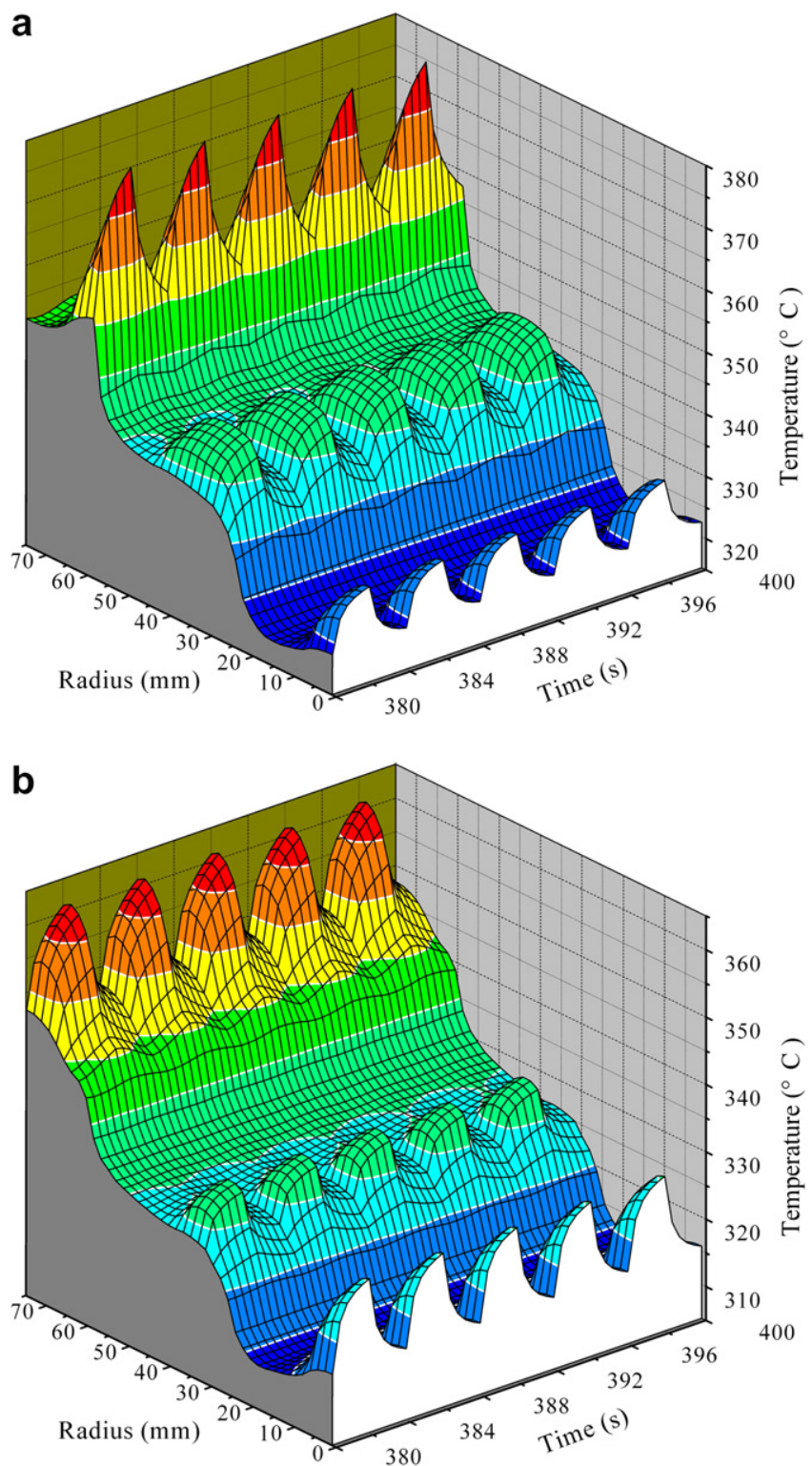

Fig. 14. Instantaneous temperatures across the radius (a) original design; (b) optimal design. 


\subsection{Tunnel air cooling}

Compared with the target temperature field, the temperature level in the middle band is still high for the optimal design. In the normal working condition, the temperature of this area is relatively low, due to the oil cooling effect from the tunnel beneath the middle band, which is indicated in Fig. 2. A loading case that includes the simulation of tunnel air cooling effect was therefore computed in order to reduce the middle band temperature. Fig. 15 gives the comparison of the instantaneous temperatures across the radius during one thermal cycle between the non-cooling case and tunnel air cooling case. The loading case of non-cooling is $P_{\mathrm{h}}=2500 \mathrm{~W}, P_{\mathrm{q}}=500 \mathrm{~W}$ and $\tau_{\mathrm{h}}=\tau_{\mathrm{q}}=2 \mathrm{~s}$. Comparably, in the tunnel air cooling case the periodic loading is $P_{\mathrm{h}}=3000 \mathrm{~W}, P_{\mathrm{q}}=1000 \mathrm{~W}$ and $\tau_{\mathrm{h}}=\tau_{\mathrm{q}}=2 \mathrm{~s}$, and a convective coefficient $h=300 \mathrm{~W} / \mathrm{mK}$ was assigned to the surface of the tunnel. Convective effect in the tunnel consumes more input energy and manifests strong influence on the overall temperature level. In order to maintain the temperature level, the input energy must be elevated in the air cooling case. The middle band temperature drops to a level lower than that of center band when combined
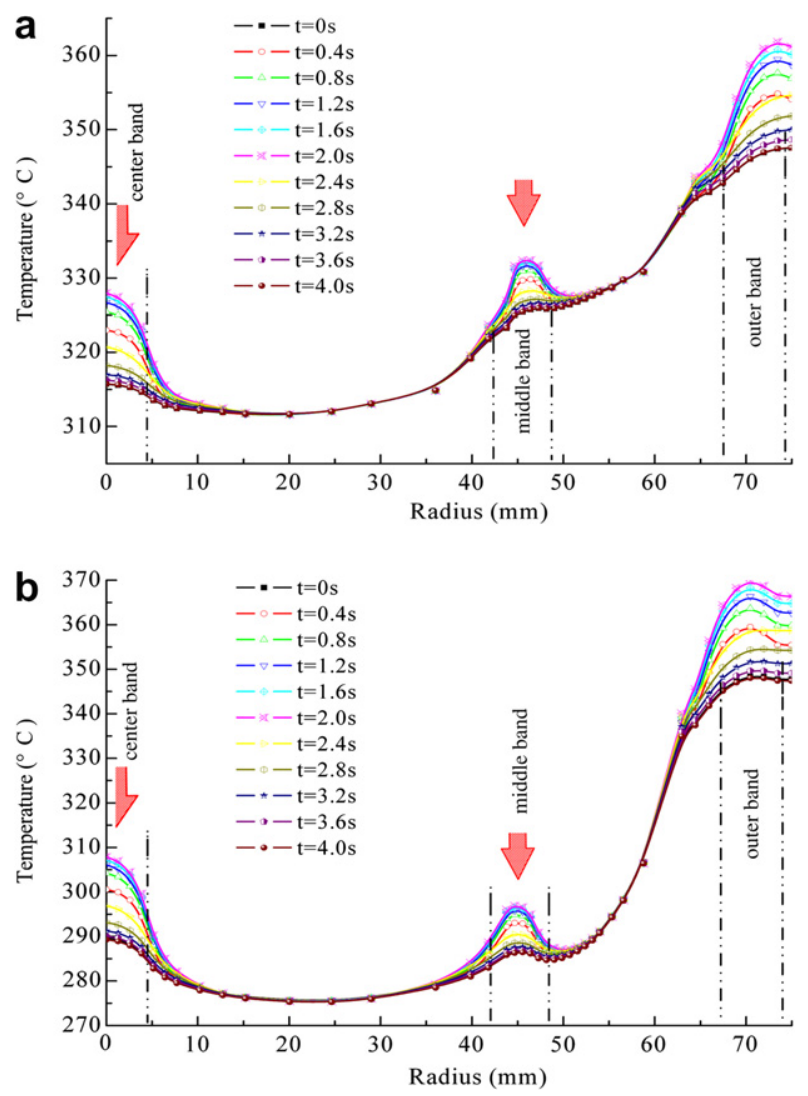

Fig. 15. Instantaneous temperatures across the radius during one thermal cycle, optimal design (a) without air cooling, in the periodic loading of $P_{\mathrm{h}}=2500 \mathrm{~W}, P_{\mathrm{q}}=500 \mathrm{~W}$ and $\tau_{\mathrm{h}}=\tau_{\mathrm{q}}=2 \mathrm{~s}(\mathrm{~b})$ air cooling at oil tunnel, where $h=300 \mathrm{~W} / \mathrm{mK}$ was assigned to the surface of the tunnel, in the periodic loading of $P_{\mathrm{h}}=3000 \mathrm{~W}, P_{\mathrm{q}}=1000 \mathrm{~W}$ and $\tau_{\mathrm{h}}=\tau_{\mathrm{q}}=2 \mathrm{~s}$.

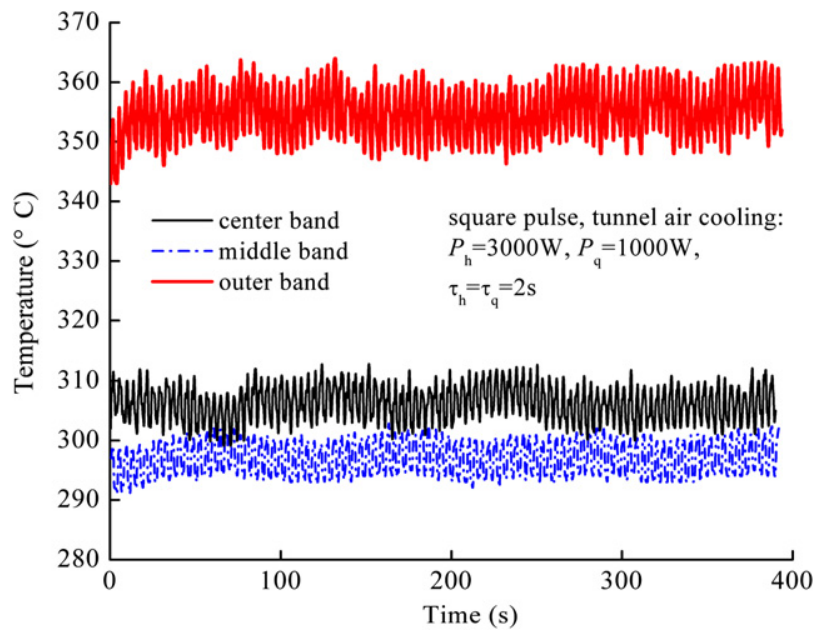

Fig. 16. Experiments incorporated with tunnel air cooling.

with tunnel air cooling, resulting a temperature distribution similar to the target, as can be seen in Fig. 15b. This loading case and temperature distribution is verified by the experiment, which is illustrated in Fig. 16. Related experiments can also be found in Fig. 6 of the accompanying paper [1].

Fig. 15 also indicates a state of dynamic equilibrium, manifested as the superposition of the curves $t=4.0 \mathrm{~s}$ and $t=0 \mathrm{~s}$. The maximum temperature difference was found between $t=2.0 \mathrm{~s}$ and $t=0 \mathrm{~s}$ ( or $t=4.0 \mathrm{~s}$ ).

\subsection{Flexibility in the design of temperature field}

The advantage of laser induced thermal fatigue lies in the flexibility and versatility in the temperature field design. Fig. 17 shows the great diversity in the temperature field of the top surface when the laser was shaped into apparent different intensity distribution. The flexibility means one can design the intensity field for the shaped laser according to specific temperature field requirement.

\section{Conclusions}

The numerical method manifests strong reliability and flexibility in the design of spatial intensity distribution of shaped laser in order to obtain the desired temperature field. The numerical method can deal with the complicated problems which cannot be solved by simple mathematical models or experimental procedures. Meanwhile, the validated numerical model in the present paper can serve as a kind of virtual experimental vehicle, which may reduce the cost and period of experiments, and makes the thermal fatigue simulation tests more controllable and predictable.

This is the first try of thermal fatigue simulation on pistons induced by the shaped high power laser. Although the 

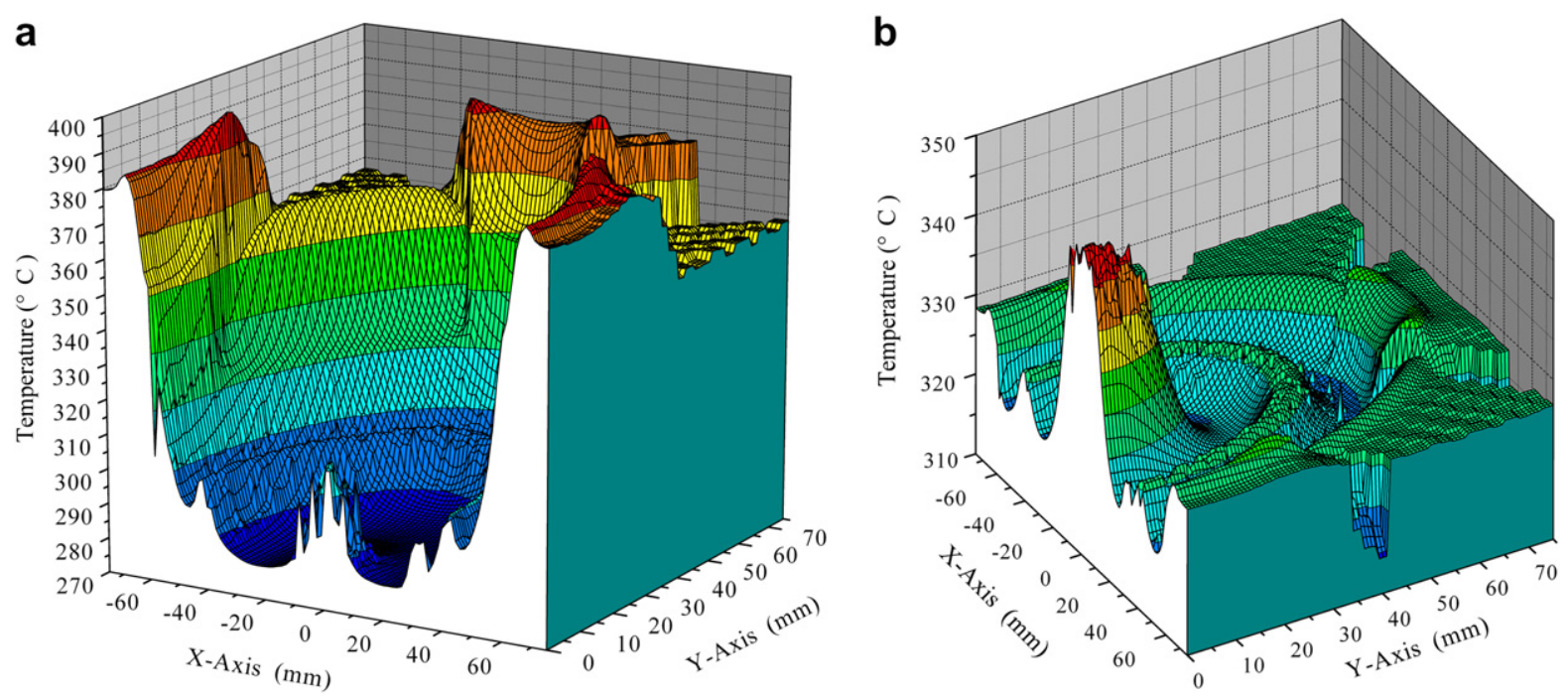

Fig. 17. Flexibility in the design of temperature field with the shaped high power laser (a) when transformed into the intensity field of $\delta_{1}=28, \delta_{2}=20$, $\delta_{3}=30$, and $r_{1}=4, r_{2}=42, r_{3}=47, r_{4}=67$ and $r_{5}=74 \mathrm{~mm}$, and incorporated with tunnel air cooling; (b) when transformed into the intensity field of $\delta_{1}=30, \delta_{2}=20, \delta_{3}=28$, and $r_{1}=15, r_{2}=40, r_{3}=48, r_{4}=65$ and $r_{5}=73 \mathrm{~mm}$.

temperature field on the top surface of the piston may not be the same as the present case [23], the shaped high power laser did show good controllability and designability in the spatial and temporal domain with the aid of DOE, therefore, it could reach the target temperature field. The shaped high power laser is a competent heat source in the thermal fatigue tests of workpieces with complex geometry.

\section{Acknowledgements}

Supports for this work from National Nature Science Foundation of China (No. 10502049) and Foundation of State Key Laboratory of Nonlinear Mechanics from Institute of Mechanics, Chinese Academy of Sciences, are gratefully acknowledged.

\section{References}

[1] H.W. Song, G. Yu, J.S. Tan, L. Zhou, X.L. Yu, Thermal fatigue on pistons induced by shaped high power laser. Part I: Experimental study of transient temperature field and temperature oscillation, Int. J. Heat Mass Transfer, in press, doi:10.1016/j.ijheatmasstransfer. 2007.04.035.

[2] D. Bauerle, Laser Processing and Chemistry, Springer-Verlag, Berlin, Heidelberg, 2000.

[3] F.M. Dickey, Laser beam shaping, Opt. Photon. News (2003) 31-34.

[4] Y. Chen, C.H. Gan, L.X. Wang, G. Yu, A. Kaplan, Laser surface modified ductile iron by pulsed Nd : YAG laser beam with twodimensional array distribution, Appl. Surf. Sci. 245 (2005) 316-321.

[5] K. Fuse, T. Hirai, T. Ushiro, T. Okada, K. Kurisu, K. Ebata, Design and performance of multilevel phase fan-out diffractive optical elements for laser materials processing, J. Laser Appl. 15 (2003) 246-254.
[6] F.M. Dickey, S.C. Holswade, Laser Beam Shaping: Theory and Techniques, Marcel Dekker Inc., New York, 2000.

[7] J.S. Tan, J.B. Di, Transient Temperature Field of Pistons, Experimental Report, China North Engine Research Institute, Datong, 2002 (in Chinese).

[8] A.M. Prokhorov, Laser Heating of Metals, IOP Publishing Ltd, New York, 1990.

[9] J.F. Ready, Industrial Applications of Lasers, Academic Press, New York, 1997.

[10] A.F.H. Kaplan, Model of the absorption variation during pulsed laser heating applied to welding of electronic $\mathrm{Au} / \mathrm{Ni}$-coated $\mathrm{Cu}$ leadframes, Appl. Surf. Sci. 241 (2005) 362-370.

[11] V. Gupta, J. Srinivasan, Heat and Mass Transfer, Tata McGraw-Hill Publishing Co. Ltd., 1978.

[12] D.J. Lewis, E.J. Chubb, H.A. Money, Factors affecting thermal stress in power plant, in: Thermal Stresses and Thermal Fatigue, Butterworth, London, 1971, pp. 324-339.

[13] H.G. Woo, H.S. Cho, Three-dimensional temperature distribution in laser surface hardening processes, Proceedings of the I MECH E Part B Journal of Engineering Manufacture, Professional Engineering Publishing, 1999, pp. 695-712.

[14] W.M. Steen, J. Mazumder, Mathematical Modelling of Laser/ Material Interactions, Final Report, European Office of Aerospace Research and Development, London, 1983.

[15] A.I. Katsamas, G.N. Haidemenopoulos, Surface hardening of lowalloy $15 \mathrm{CrNi6}$ steel by $\mathrm{CO}_{2}$ laser beam, Surf. Coat. Technol. 115 (1999) 249-255.

[16] B.Q. Xu, Z.H. Shen, J. Lu, X.W. Ni, S.Y. Zhang, Numerical simulation of laser-induced transient temperature field in filmsubstrate system by finite element method, Int. J. Heat Mass Transfer 46 (2003) 4963-4968.

[17] H. Pantsar, V. Kujanpaa, Effect of oxide layer growth on diode laser beam transformation hardening of steels, Surf. Coat. Technol. 200 (2006) 2627-2633.

[18] C. Lampa, A.F.H. Kaplan, J. Powell, C. Magnusson, The effect of process speed on energy redistribution in deep penetration $\mathrm{CO}_{2}$ laser welding, High Temp. Mater. P-Us 4 (2000) 213-225.

[19] X.E. Lin, Laser pulse heating, in: Proceedings of the 1999 Particle Accelerator Conference, New York, 1999, pp. 1429-1431. 
[20] Y. Jaluria, K.E. Torrance, Computational Heat Transfer, Series in Computational Methods in Mechanics and Thermal Sciences, Hemisphere Publishing Corporation, New York, 1986.

[21] D.B. Spalding, Heat conduction, in: P.P. Benham, R. Hoyle (Eds.), Thermal Stress, Sir Isaac Pitman \& Sons Ltd, London, 1964, pp. 323.
[22] Q. Zhang, Piston Design Handbook, China North Engine Research Institute, Datong, 1988, in Chinese.

[23] A. Weronski, T. Hejwowski, Thermal Fatigue of Metals, Marcel Dekker, Inc., New York, 1991. 